\title{
Some Generalities About Generality
}

\author{
John D. Barrow \\ DAMTP, University of Cambridge \\ Wilberforce Rd., Cambridge CB3 0WA \\ UK
}

\begin{abstract}
We survey a variety of cosmological problems where the issue of generality has arisen. This is aimed at providing a wider context for many claims and deductions made when philosophers of science choose cosmological problems for investigation. We show how simple counting arguments can be used to characterise parts of the general solution of Einstein's equations when various matter fields are present and with different spatial topologies. Applications are described to the problem of singularities, static cosmological models, cosmic no hair theorems, the latetime isotropisation of cosmological models, and the number of parameters needed to describe a general astronomical universe.
\end{abstract}

\section{Introduction}

The equations of general relativity and its extensions are mathematically complicated and their general coordinate covariance offers special challenges to anyone seeking exact solutions or conducting numerical simulations. They are nonlinear in a self-interacting (non-Abelian) way because the mediator of the gravitational interaction (the graviton) also feels the gravitational force. By contrast in an Abelian theory, like electromagnetism, the photon does not possess the electric charge that it mediates. As a result of this formidable complexity and non-linearity, the known exact solutions of general relativity have always possessed special properties. High symmetry, or some other simplifying mathematical property, is required if Einstein's equations are to be solved exactly. General solutions are out of reach.

This 'generality' problem has been a recurrent one in relativistic cosmology from the outset in 1916 when Einstein 1 first proposed a static spatially homogeneous and isotropic cosmological model with non-Euclidean spatial geometry in which gravitationally attractive matter is counter-balanced by a positive cosmological constant. This solution turned out to be unstable [2. Subsequently, the appearance of an apparent 'beginning' and 'end' to simple expanding universe solutions led to a long debate over whether these features were also unstable artefacts of high symmetry or special choices of matter in the known 
cosmological solutions, as Einstein thought possible. The quest to decide this issue culminated in a new definition of such 'singularities' which allowed precise theorems to be proved without the use of special symmetry assumptions. In fact, by using the geodesic equations, their proofs made no use of the Einstein equations [3. Special solutions of Einstein's equations, like the famous Gödel metric [4] with its closed timelike curves, also provoked a series of technical studies of whether its time-travelling paths are a general feature of solutions to Einstein's equations, or just isolated unstable examples. In the period 1967-1980 there was considerable interest in determining whether the observed isotropy of the microwave background radiation could be explained because it appeared to be an unstable property of expanding universes [5]. The mechanism of 'inflation', first proposed in 1981 by Guth [6], provided a scenario in which this conclusion could be reversed, and isotropy could be a stable (or asymptotically stable) property of expanding universe solutions, by widening the allowed conditions on the allowed forms of matter that could dominate the expansion dynamics of the very early universe $[7,[8$. Just to show how knowledge, fashion, and belief change, the requirements on the density, $\rho$, and pressure, $p$, of matter content needed for inflation to occur $(\rho+3 p<0)$ are exactly the opposite of those assumed $(\rho+3 p>0)$ in the principal singularity theorems of Penrose and Hawking [3, 9] in order to establish sufficient conditions for a singularity (at least one incomplete geodesic) to have occurred in our past.

In the study of differential equations, an exact solution is called stable if small perturbations remain bounded as time increases; it is called asymptotically stable if the perturbations die away to zero with increasing time. Our solar system is dynamically stable but not asymptotically stable. Another useful pair of definitions are those introduced by Hawking [10 in 1971, who uses the same word in a technically different way. He defines a 'stable' or 'open' property of a dynamical system to be one that occurs from an open set (rather than merely a single point) in initial data space. However, it is possible for a property of a cosmological model to be stable but be of no physical interest: it is a necessary but not a sufficient property for physical relevance because the property in question could be stable only in open neighbourhoods of initial data space describing universes with other highly unrealistic properties (contraction or extreme anisotropy, for example). A 'generic' or 'open dense' property will be one that occurs near almost every initial data set (that is, it is open dense on the space of all initial data). A sufficient condition for a stable property to be of physical interest is that it is generic in this sense [11.

In this chapter we will discuss approaches to the problem of assessing generality and some of the results that arise in typical and topical cosmological problems. We will try to avoid significant technicalities. There is a deliberate emphasis upon fundamental questions of interest to philosophers of science rather than upon the astrophysical complexities of the best-fit cosmological models or the galaxy of inflationary universe models. Attention will be focussed on classical general relativity; aspects of quantum cosmology will be treated in other chapters. 


\section{General relativistic and Newtonian cosmology}

General relativity is a much larger theory than Newtonian gravity. It has ten symmetric metric potentials, $g_{a b}$, instead of one Newtonian gravitational potential, $\Phi$, and ten field equations (Einstein's equations) instead of a single one (Poisson's equation to determine them from material content of space and time. Newtonian gravity has a fixed time and a fixed space geometry which is usually taken to be a monotonous linear time plus a 3-d Euclidean space (although another fixed curved space could be used simply by using the appropriate $\nabla^{2}$ operator in Poisson's equation).

Despite appearances, Newton's theory is not really complete and Newtonian cosmology is not a well-posed theory [12, 13. Unlike general relativity, it contains no propagation equations for the shear distortion and the formulation of anisotropic cosmological models requires these to be put in by hand. As a result the Newtonian description of an isotropic and homogeneous cosmology looks exactly like general relativity [14] because these shear degrees of freedom are necessary absent. This feature manifests itself in results for the general asymptotic behaviour of the Newtonian n-body problem in the unbound (expanding) case. Rigorous results can be obtained for the moment of inertia (or radius of gyration), or rotation of the total finite mass of n-bodies, but not for its shape [15], [12].

All solutions of Einstein's equations describe entire universes. The relative sizes of the two theories means that infinitely many of these general relativistic solutions possess no Newtonian counterpart. However, there are also Newtonian 'universes' which have no counterpart in general relativity. For example there are shear-free Newtonian solutions with expansion and rotation: these cannot exist in general relativity [16]. More striking, there exist solutions of the Newtonian n-body problem (for $n>4$ ) in which a system of point particles expands to infinite size in finite time, undergoing an infinite number of oscillations in the process 17. For example, two counter-rotating binary pairs, all of equal mass, with a lighter particle oscillating between their centres along a line perpendicular to their orbital planes can expand to infinite size as a result of an infinite number of recoils in a finite time! This is only possible because Newtonian point particles can get arbitrarily close to one another and so the $1 / r^{2}$ forces between them an become arbitrarily large. In general relativity this cannot happen. When two point particles of mass $M$ approach closer than $4 G M / c^{2}$ an event horizon forms around them. This is a simple example of a form of 'cosmic censorship' that saves us from the occurrence of an actual observable infinity, in Aristotle's sense [18, locally. In general relativity there is evidence that under broad conditions there is a maximum force, equal to $c^{4} / G$, [19] as well as the more fundamental a maximum velocity for information transfer, $c$ : neither of these relativistic limits of velocity and force strength exist in Newtonian theory. 


\section{Generality - some historic cases}

There have been a succession of cosmological problems where particular solutions were found with striking properties that required further analysis to determine whether those properties were general features of cosmological solutions to the Einstein equations.

\subsection{Static universes}

The first isotropic and homogeneous cosmological model found by Einstein [1] was a static universe with zero-pressure matter, positive cosmological constant and a positive curvature of space. Subsequently, this solution was shown to be unstable when it was perturbed within the family of possible isotropic and homogeneous solutions of Einstein's equations by Eddington and implicitly by Lemaitre [20] who found the general solutions of which Einstein's universe was a particular, and clearly unstable, case. These demonstrations led to the immediate abandonment of the static universe and, in Einstein's case, of the cosmological constant as well [21. It turns out that this stability problem is more complicated than it appears and has only been completely explored, when other forms of matter are present, quite recently. The static universe is only unstable against small inhomogeneous perturbations on scales exceeding the Jeans length when $p / \rho<1 / 5$. When $1 \geq p / \rho>1 / 5$, the Jeans length for the inhomogeneities exceeds the size of the universe and so the instability does not become Jeans unstable and amplify in time [2].

\section{$3.2 \quad$ Singularities}

There is a long and interesting history of attempts to interpret and avoid 'singularities' in the cosmological solutions of Einstein's equations. In the first expanding solutions with zero-pressure matter found by Friedmann [22, it appeared that there was a necessary beginning to the expansion with infinite density at a finite time in the past and there could (in spatially closed cases) also be an apparent end to the universe at a finite time in the future. One response to these infinities, particularly by Einstein, was to question whether they would remain if the family of solutions was widened. First, Einstein asked whether the addition of pressure would resist the compression to infinite density. Lemaittre showed that adding pressure actually made the problem worse by hastening the appearance of the infinite density [23. The reason is a relativistic one. Whereas in Newtonian physics any pressure resists gravitational compression, in relativity pressure also gravitates because it is a form of energy (and so has an equivalent mass via ' $E=m c^{2}$ ') and increases the compression (see also [24]). Next, Einstein wondered whether it was the perfect isotropy of the expanding universe solutions that was responsible. If anisotropy was allowed then perhaps the compression would be defocussed and the singularity avoided. Again, Lemaître was easily able to show that simple anisotropic universes have the same types of singularity and they are approached quicker than 
in the isotropic case [23].

These investigations by Lemaitre amounted to tests of the stability of the singularity occurrence within different wider sets of initial data. Many cosmologists were convinced by these examples that singularities were ubiquitous in these types of cosmology unless new forms of matter could be found which resisted compression to infinite density. One such material was the C-field of the steady state theory, introduced by Hoyle to describe 'continuous creation' of matter in a de Sitter universe that avoided the high-density singularities of the Friedmann-Lemaître models. However, all its null geodesics are past incomplete and so it is technically singular 25] (a feature that has been recently rediscovered in another context [26]).

Later, in the period 1957-1966, a different approach was pursued for a while by members of Landau's school in Moscow, notably by Khalatnikov and Lifshitz [27. Initially, they set out to show that singularities did not occur in the general solution of the Einstein equations. Their argument, which was incorrect, was that because singularities arose in solutions that were not general (like the Friedmann solutions or the anisotropic Kasner universes) they would appear in the general solution. The circumstantial evidence for this conclusion was the belief that the singularity arising in these cosmological solutions was just a singularity in the coordinate system used to describe the dynamics and so was unphysical (as is the 'singularity' that arises at the North Pole of the Earth where the meridians intersect in standard mapping coordinates). When it occurred you could change to a new set of coordinates until they too became singular (as they always did) and so on ad infinitum. Unfortunately, it is important to investigate what happens in the limit of this process: a true physical singularity remains as became increasingly clear when the problem was subjected to a different sort of analysis. The singularity theorems of Hawking and Penrose [3] were able to define sufficient conditions for the formation of a singularity by adopting a definition of a singularity as an inextendible path of a particle or light-ray in spacetime. These theorems made no reference to special symmetries or the subtleties of coordinate choices. Singularities were where time ran out: part of the edge of spacetime 28. It remained to be shown that these endpoints were caused by infinities in physical quantities. To some extent this can be done but the full story is by no means complete, even now. These theorems ended the argument about whether cosmological singularities were physically real and general and later work by Belinskii, Khalatnikov and Lifshitz refocussed upon finding the general behaviour near a physical singularity [29].

It is important to stress that the singularities are theorems, not theories. They give sufficient conditions for singularities so if their assumptions are not all met this does not mean that there is no inevitable singularity, merely that no conclusions can be drawn. The interesting historical aspect which we signalled in the introduction is that the sufficient conditions generally included the requirement that the matter content of the universe obeys $\rho+3 p>0$. We no longer believe this inequality holds for all matter sources. Indeed, the observations that the universe is accelerating could be claimed to show that the assumption that $\rho+3 p>0$ is false. 


\subsection{Isotropisation}

After the discovery in 1967 of the high level of isotropy in the CMB temperature distribution [30] there was a long effort to explain why this was the case. Up until then cosmologists had assumed an isotropic and homogeneous background universe and regarded the presence of small inhomogeneities (like galaxies) as the major mystery requiring a simple explanation. The discovery of the CMB isotropy placed created a new perspective in which it was the high isotropy and uniformity of the assumed background universe that was the major mystery. A new approach, proposed by Misner and dubbed 'chaotic cosmology' sought to show that general cosmological initial conditions would end up leading to an isotropically expanding universe after more than about 10 billion years [5]. This programme had an interesting methodological aspect. If it could be shown that almost all initial conditions (subject to some weak conditions of physical reasonableness) would lead to isotropic universe then observations of the isotropy level could not tell us anything about the initial conditions: memory of them would have been erased by the expansion.

In studying whether this idea could work it was again the issue of generality that was crucial. It was asking whether isotropically expanding universes were stable or even asymptotically stable attractors at late times. Two types of analysis were performed. The first just asked whether anisotropic cosmologies would approach isotropy at late times if they just contain zero-pressure matter and radiation. The second, which Misner proposed, was to ask what happened if dissipative stresses could arise because of the presence of collisionless particles, like neutrinos and gravitons, at particular epochs in the very early universe. Perhaps large initial anisotropies could be damped out by these dissipative processes, leaving an isotropically expanding universe?

Several interesting approaches to these questions were developed. On the physical level Barrow and Matzner [31] showed that the chaotic cosmology philosophy could not work in general because the dissipation of anisotropies and inhomogeneities must produce heat radiation, in accord with the second law of thermodynamics. The earlier dissipation occurred the larger entropy per baryon produced and the observed entropy per baryon today (of about $10^{9}$ ) placed a prohibitively strong bound on how much anisotropy could have been damped out during the history of the universe. The presence of particle horizons with proper radii proportional to $t$ in the early universe also placed a major constraint on the damping of any large scale inhomogeneities by causal processes. Misner 32 attempted to circumvent this by discovering the remarkable possibility that spatially homogeneous universes of Bianchi type IX (dubbed the 'Mixmaster' universe because of this property) could potentially allow light to travel around the universe arbitrarily often on approach to $t=0$ as a result of their chaotic dynamics. Unfortunately, this horizon removal mechanism was ineffective in practice because of the improbability of the horizonless dynamical configurations and the fact that only about 20 chaotic oscillations of the scale factor could have occurred between the Planck time $\left(10^{-43} \mathrm{~s}\right)$ and the present [33. There was also the concern that if general relativistic cosmology was a 
well-behaved initial-value problem then one could always concoct anomalously anisotropic universe today that could be evolved back to their initial conditions at any arbitrary early time [34. These would provide counter examples to the chaotic cosmology scheme although one has to be careful with this argument because the counter examples could all have physically impossible initial conditions, and in fact they often do 35.

In 1973 Collins and Hawking [36] carried out some interesting stability analyses of isotropic universes to discover if isotropy was a stable property of homogeneous initial data. The results found were widely discussed and reported at a semi-popular level but needed to be treated cautiously because of the fine detail in the theorems. They reported that for ever-expanding universes isotropic expansion was 'unstable' but if attention was narrowed to spatially flat initial data with zero-pressure matter then isotropy was 'stable'. The cosmological constant was assumed to be zero. The definition of stability used was in fact asymptotic stability and so the proof that isotropy was unstable just meant that anisotropies did not tend to zero as $t \rightarrow \infty$. In fact, closer analysis showed that in general $\sigma / H \rightarrow$ constant in open universes (and it is impossible for $\sigma / H$ to grow asymptotically) with $\rho+3 p>0$. This means that isotropy is stable, although not asymptotically stable, [37, 38]. The other technicality, is that this result is a consequence the fact that these open universe become vacuum (or spatial curvature dominated) at late times. The behaviour of the anisotropy it therefore an asymptotic property of vacuum cosmologies and doesn't tell us anything about the past history of the universe at redshifts $z>z_{c}$, where $z_{c}<O(1)$ is the redshift where the expansion becomes curvature dominated. These stability results therefore did not help us understand the sort of initial data that could give rise to high isotropy after about 10 billion yeas of expansion.

\subsection{Cosmic no-hair theorems}

Amid all this interest in explaining the isotropy of the universe there was one prescient approach by Hoyle and Narlikar 39] that predated the discovery of the high isotropy of the CMB. In 1963 they pointed out that in the standard big bang model the isotropy and average uniformity of the universe was a mystery but in the steady state universe it would be naturally explained. To support this claim they showed that the de Sitter universe is stable against scalar, vector and tensor perturbations. Thus, a steady state universe, described by the de Sitter metric of general relativity would always display high isotropy and uniformity. In fact, had they only known it they could have predicted that the only spectrum of perturbations consistent with the steady state universe is the constant curvature spectrum with constant (small) metric perturbations on all scales that is observed with high accuracy today - although ironically via perturbations in the CMB whose existence the steady state model could not explain). Any departure from this spectrum, with $\delta \rho / \rho \propto L^{-2}$ on length scale $L$ would either create divergent metric potential perturbations as $L \rightarrow \infty$ or $L \rightarrow 0$.

The idea of the inflationary universe provided a new type of explanation for 
the observed isotropy and uniformity of the universe from general initial conditions, but with one important difference from past expectations - the isotropy and homogeneity was predicted to be local. The inflationary universe theory proposed that there was a finite interval of time, soon after the apparent beginning of the expansion (typically at $\sim 10^{-35} \mathrm{~s}$ in the original conception of the theory), when the expansion of the universe would accelerate due to the presence of very slowly evolving scalar fields of the sort that appeared in new theories of high-energy physics. These would contribute stresses with $\rho+3 p<0$ and cause the expansion scale factor to accelerate. When this occur the expansion rapidly approaches isotropy. Anisotropies fall off very rapidly and isotropic expansion is asymptotically stable. In the most likely scenario, where $p=-\rho$, the expansion behaves temporarily like Hoyle's steady state universe and grows exponentially in time. However, the inflation needs to an end, and this can happen if the scalar fields responsible decay into ordinary particles and radiation with $\rho+3 p>0$. When this happens the usual decelerating expansion is resumed but with anisotropies so diminished in amplitude that they remain imperceptibly small late late times [7, 8,

Inflation works by taking a small patch of the universe that is small enough for light signals to cross it at an early time $t_{I}$ and expanding it so dramatically (exponentially in time) that it grows larger than the entire visible universe today in the short period during which inflation occurs. Thus the isotropy and high uniformity of the visible universe today is a reflection of the fact that it is the expanded image of a region that was small enough to be coordinated by light-like transport processes and damping when inflation occurred. If inflation had not occurred, and the expansion had merely continued along its standard decelerating trajectory then the initially smooth and isotropic region would not have expanded significantly by the present time, $t_{0}$. Here is a simple calculation of how this happens.

Suppose the preset temperature of the CMB is $T_{0}=3 K$ and when inflation occurred is was $T_{I}=3 \times 10^{28} \mathrm{~K}$. Then, since $T \propto a^{-1}$, the scale factor has increased by a factor of $T_{I} / T_{0}=10^{28}$. At time $t_{I}$, the horizon size is equal to $d\left(t_{I}\right)=2 c t_{I}$ where $t_{I} \simeq 10^{-35} \mathrm{~s}$, so a horizon-sized region of size $2 c t_{I}$ at $t_{I}$ would only have expanded to a size $2 \times 3 \times 10^{10} \mathrm{cms}^{-1} \times 10^{-35} \mathrm{~s} \times 10^{28}=$ $6 \times 10^{3} \mathrm{~cm}$ by the present day. This is not of any relevance for explaining isotropy and uniformity over scales of order $c t_{0} \sim 10^{28} \mathrm{~cm}$ today. However, suppose inflation occurs at $t_{I}$ and inflates the expansion scale factor by a factor of $e^{N}$. We will now be able to enlarge the causally connected region of size $2 c t_{I}$ up to a scale of $e^{N} \times 6 \times 10^{3} \mathrm{~cm}$. This will exceed the size of the visible universe today if $e^{N} \times 6 \times 10^{3} \gtrsim 10^{28}$. This is easily possible with $N>60$. If the expansion is exponential with $a(t) \propto e^{H t}$ then we only need inflation to last from about $10^{-35} s$ until $10^{-33} s$ in order to effect this. The regularity of the universe is therefore explained without any dissipation taking place. A very tiny smooth patch is simply expanded to such an extent that its smooth and isotropic character is reflected on the scale of the entire universe today. It is very likely (just as it is more likely that a randomly chosen positive integer will be a very large one) that the amount of inflation that occurred will be much large 
than 60 e-folds. Yet, the result is to predict that the universe will be uniform on the average out to the inflated scale $e^{N} \times 6 \times 10^{3}$ but may be rather non-uniform if we could see further. In some variants of the theory many other fundamental features of the universe (values of constants of Nature, space dimensions. laws of physics) are different beyond the inflated scale as well. While there have always been overly positivistic philosophers who have cautioned against simply assuming that the unobserved part of the (possibly infinite) universe is the same on average as the observed part, this is the first time there has been a positive prediction that we should not expect them to be the same.

The result of a sufficiently long period of accelerated expansion is to drive the local expansion dynamics of the universe is to drive the expansion dynamics towards the isotropic de Sitter expansion, with asymptotic form of the metric of the form $(\alpha, \beta=1,2,3)[8$

$$
\begin{aligned}
d s^{2} & =d t^{2}-g_{\alpha \beta} d x^{\alpha} d x^{\beta} \\
g_{\alpha \beta} & =\exp [2 H t] a_{\alpha \beta}(\mathbf{x})+b_{\alpha \beta}(\mathbf{x})+\exp [-H t] c_{\alpha \beta}(\mathbf{x})+\ldots,
\end{aligned}
$$

where $H$ is the constant Hubble rate, with $3 H^{2}=\Lambda$, and $a_{\alpha \beta}(\mathbf{x}), b_{\alpha \beta}(\mathbf{x})$ and $c_{\alpha \beta}(\mathbf{x})$ are arbitrary symmetric spatial functions. The Einstein equations allow only two of the $a_{\alpha \beta}$ and two of the $c_{\alpha \beta}$ to be freely specifiable and all the $b_{\alpha \beta}$ are determined by them. Thus there are four independently arbitrary spatial functions specifying the solution on a spacelike surface of constant in vacuum. Notice the spatial functions $a_{\alpha \beta}(\mathbf{x})$ at leading order in the metric $(H$ is a constant though). This is why the metric only approaches de Sitter locally, exponentially rapidly inside the event horizon of a geodesically moving observer. If radiation is black body $(p=\rho / 3)$ is added then a further 4 arbitrary spatial functions are required (three for the normalised 4-velocity components and one for the density) and

$$
\begin{aligned}
\rho & \propto \exp [-4 H t], \\
u_{0} & \rightarrow 1, u_{\alpha} \propto \exp [H t] c_{\alpha ; \beta}^{\beta}, \\
c_{\alpha}^{\alpha} & =0 .
\end{aligned}
$$

Hence, we see that the 3 -velocity $V^{2}=u_{\alpha} u^{\alpha}$ tends to a constant as $t \rightarrow \infty$. The asymptotic state is therefore de Sitter plus a constant (or 'tilted') velocity field which affects the metric at third order (via $c_{\alpha \beta}(\mathbf{x})$ ). This is easy to understand physically if we consider a large rotating eddy that expands with the universe and has angular velocity $\omega=V a^{-1}$. Its angular momentum is $M a^{2} \omega \propto\left(\rho a^{3}\right) a^{2}\left(V a^{-1}\right)$ and this is conserved as the universe expands. since the radiation density falls as $\rho \propto a^{-4}$, we have $V$ constant as $a \rightarrow \infty$.

The number of free spatial functions specifying this asymptotic solution is 8 in the case with radiation (and the same holds when any other perfect fluid matter is present). In the next section we will show that this is characteristic of a part of the general solution of Einstein's equations. 
In conclusion we see that a finite period of accelerated expansion is able to drive the expansion towards isotropy from a very large class of initial conditions (not all initial conditions, since the universe must not, for example recollapse before a period of accelerated expansion begins). We have just discussed the most extreme form of accelerated expansion with constant Hubble expansion rate, $H$, and $a \propto \exp [H t]$ but similar conclusions hold for power-law inflation, with $a \propto t^{n}, n>1$, and intermediate inflation, with $a \propto \exp \left[A t^{n}\right]$, with $A>0$, and $0<n<1$ constants. The key conceptual point is that explains the present isotropy without dissipating initial anisotropies in the way that the chaotic cosmology programme imagined and so it evades the Barrow-Matzner entropy per baryon constraint [31. Instead it drives the initial inhomogeneities far beyond the visible horizon today and the stress driving the acceleration dominates over all forms of anisotropy at large expansion volumes and times. The earlier analyses of the stability of isotropic expansion by Collins and Hawking, and others, [36, had restricted attention to forms of matter in the universe with $\rho+3 p>0$ and always assumed $\Lambda=0$ because there was no reason to think otherwise at that time. As a result, they had excluded the possibility of accelerated expansion which can solve the isotropy problem without any dissipation occurring if it can arise for a finite period of time in the early universe .

\subsection{The initial value problem}

The attempts to explain the isotropy of the universe from arbitrary initial conditions gave rise to another interesting perspective that is worth highlighting. General relativity is an initial value problem an so for 'well-behaved' cosmological solutions this means that the present state of the universe described by any solution of Einstein's equations is a continuous function of some 'initial data' at any past time. In a technical sense it might appear that given any state of the universe today - highly anisotropic, for example - then there exists some initial data set that evolves to give that state regardless of the action of any damping effects. Hence, there could never be a theory that could explain the actual state of the universe today as the result of evolution from any (or almost any) initial conditions. The problem with this argument is that the initial conditions that do evolve to counter-factual cosmological states at late times may arise only from initial data states that are completely unphysical in some respect [35]. Take a simple example of a Bianchi type I anisotropic universe. The anisotropy energy density and radiation energy density fall as

$$
\begin{aligned}
& \sigma^{2}=\sigma_{0}^{2}(1+z)^{6}, \\
& \rho_{\gamma}=\rho_{\gamma 0}(1+z)^{4} .
\end{aligned}
$$

We can choose values of the constant $\sigma_{0}^{2}$ so that the universe's expansion is dominated by anisotropy today - just pick $\sigma_{0}^{2}=\rho_{\gamma 0} \simeq 10^{-34} \mathrm{gmcm}^{-3}$ to specify the initial data. However, if we run this apparent counter-example back to the time when the radiation temperature is $T_{p l} \simeq 10^{32} K=3\left(1+z_{p l}\right)$ when its energy density equals the Planck density, $10^{94} \mathrm{gmcm}^{-3}$, we require the anisotropy 
energy density to be $10^{64}$ times larger than the Planck energy density at that time - a completely unphysical situation. Alternatively, if we had taken the anisotropy energy density to be the Planck density at $z_{p l}$ then we have the strange initial condition that the radiation density is $10^{64}$ times smaller despite all forms of energy being in quantum gravitational interaction at that time.

This is a (deliberately) dramatic example but the basic problem with the argument is one that one can find with other arguments regarding the generality of more complicated outcomes in cosmology. For example, there have been claims (and claims to the contrary) that inflation is not generic for Friedmann universes containing scalar fields with a quadratic self-interaction potential [40. The claim is based on using the Hamiltonian measure in the phase space for the dynamics to show that the bulk of the initial data measure is for solutions which don't inflate. This type of initial data corresponds to solutions with huge

initial kinetic term $\left(\dot{\phi}^{2}\right)$ which dominate the potential $V(\phi)=m^{2} \phi^{2}$ by a huge factor so that the potential never comes to dominate the dynamics by any prespecified epoch. However, this doesn't look very natural because it requires the two forms of energy density to differ by an enormous factor when one of them equals the Planck energy density (above which we know nothing about what happens since general relativity, quantum mechanics and statistical mechanics all break down). The better course is to not let any energy density exceed the Planck value but (surprisingly) this appears to be controversial.

\section{Naive function counting}

There have been several attempts to reduce the description of the astronomical universe to the determination of a small number of measurable parameters. Typically, these will be the free parameters of a well defined cosmological model that uses the smallest number of constants that can provide a best fit to the available observational evidence. Specific examples are the popular characterisations of cosmology as a search for 'nine numbers' 41, 'six numbers' 42, or the six-parameter minimal $\Lambda C D M$ model used to fit the WMAP [43] and Planck data sets [44. In all these, and other, cases of simple parameter counting there are usually many simplifying assumptions that amount to ignoring other parameters or setting them to zero; for example, by assuming a flat Friedmann background universe or a power-law variation of density inhomogeneity in order to reduce the parameter count and any associated degeneracies. The assumption of a power-law spectrum for inhomogeneities will reduce a spatial function to two constants, while the assumption that the universe is described by a Friedmann metric plus small inhomogeneous perturbations both reduces the number of metric unknowns and converts functions into constant parameters. In this paper we are going provide some context for the common minimal parameter counts cited above by determining the total number of spatial functions that are needed to prescribe the structure of the universe if it is assumed to contain a finite number of simple matter fields. We are not counting fundamental constants of physics, like the Newtonian gravitation constant, the coupling 
constants defining quadratic lagrangian extensions of general relativistic gravity, or the 19 free parameters that define the behaviours of the 61 elementary particles in the standard 3-generation $U(1) \times S U(2) \times S U(3)$ model of particle physics. However, there is some ambiguity in the status in some quantities. For example, as to whether the dark energy is equivalent to a true cosmological constant (a fundamental constant), or to some effective fluid or scalar field, or some other emergent effect [45] Some fundamental physics parameters, like neutrino masses, particle lifetimes, or axion phases, can also play a part in determining cosmological densities but that is a secondary use of the cosmological observable. Here we will take an elementary approach that counts the number of arbitrary functions needed to specify the general solution of the Einstein equations (and its generalisations).This will give a minimalist characterisation that can be augmented by adding any number of additional fields in a straightforward way. We will also consider the count in higher-order gravity theories as well as for general relativistic cosmologies. We enumerate the situation in spatially homogeneous universes in detail so as to highlight the significant impact of their spatial topology on evaluations of their relative generality.

Let us move on to a more formal discussion of how to specify the generality of solutions to Einstein's equations by counting the number of free functions (or constants) that a given solution or approximate solutions contains. In view of the constraint equations and coordinate covariances of the theory this requires a careful accounting.

The cosmological problem can be formulated in general relativity using a metric in a general synchronous reference system [46. Assume that there are $F$ matter fields which are non-interacting and each behaves as a perfect fluid with some equation of state $p_{i}\left(\rho_{i}\right), i=1, \ldots F$. They will each have a normalised 4 -velocity field, $\left(u_{a}\right)_{i}, a=0,1,2,3$. These will in general be different and noncomoving. Thus each matter field is defined on a spacelike surface of constant time by 4 arbitrary functions of three spatial variables, $x^{\alpha}$ since the $u_{0}$ components are determined by the normalisations $\left(u_{a} u^{a}\right)_{i}=1$. This means that the initial data for the $F$ non-interacting fluids are specified by $4 F$ functions of three spatial variables. If we were in an $N$-dimensional space then each fluid would require $N+1$ functions of $N$ spatial variables and $F$ fluids would require $(N+1) F$ such functions to describe them in general.

The 3-d metric requires the specification of $6 g_{\alpha \beta}$ and $6 \dot{g}_{\alpha \beta}$ for the symmetric spatial $3 \times 3$ metric in the synchronous system but these may be reduced by using the 4 coordinate covariances of the theory and a further 4 can be eliminated by using the 4 constraint equations of general relativity. This leaves 4 independently arbitrary functions of three spatial variables [46] which is just twice the number of degrees of freedom of the gravitational spin-2 field. The general transformation between synchronous coordinate systems maintains this number of functions [46. This is the number required to specify the general vacuum solution of the Einstein equations in a 3 -dimensional space. In an $N$ dimensional space we would require $N(N+1)$ functions of $N$ spatial variables to specify the initial data for $g_{\alpha \beta}$ and $\dot{g}_{\alpha \beta}$. This could be reduced by $N+1$ coordinate covariances and $N+1$ constraints to leave $(N-2)(N+1)$ inde- 
pendent arbitrary functions of $N$ variables [47]. This even number is equal to twice the number of degrees of freedom of the gravitational spin-2 field in $N+1$ dimensional spacetime .

When we combine these counts we see that the general solution in the synchronous system for a general relativistic cosmological model containing $F$ fluids requires the specification of $(N-2)(N+1)+F(N+1)=(N+1)(N+F-2)$ independent functions of $N$ spatial variables. If there are also $S$ non-interacting scalar fields, $\phi_{j}, j=1, . ., S$, present with self interaction potentials $V\left(\phi_{j}\right)$ then two further spatial functions are required $\left(\phi_{j}\right.$ and $\left.\dot{\phi}_{j}\right)$ to specify each scalar field and the total becomes $(N+1)(N+F-2)+2 S$. For the observationally relevant case of $N=3$, this reduces to $4(F+1)+2 S$ spatial functions.

For example, if we assume a simple realistic scenario in which the universe contains separate baryonic, cold dark matter, photon, neutrino and dark energy fluids, all with separate non-comoving velocity fields, but no scalar fields, then $F=5$ and our cosmology needs 24 spatial functions in the general case. If the dark energy is not a fluid, but a cosmological constant with constant density and $u_{i}=\delta_{i}^{0}$, then the dark energy 'fluid' description reduces to the specification of a single constant, $\rho_{D E}=\Lambda / 8 \pi G$, rather than 4 functions and reduces the total to 21 independent spatial functions. However, if the cosmological constant is an evolving scalar field then we would have $F=4$ and $S=1$, and now 22 spatial functions are required. Examples of full function asymptotic solutions were found for perturbations around de Sitter space-time by Starobinsky [8], the approach to 'sudden' finite-time singularities [48 by Barrow, Cotsakis and Tsokaros [49], and near quasi-isotropic singularities with $p>\rho$ 'fluids' by Heinzle and Sandin $[50$.

These function counts of 21-24 should be regarded as lower bounds. They do not include the possibility of a cosmological magnetic field or some other unknown matter fields. They also treat all light $(<<1 \mathrm{MeV})$ neutrinos as if they are identical (heavy neutrinos can be regarded as CDM if they provide the largest contribution to the matter density but if they are not responsible for the dominant dark matter then they should be counted as a further contribution to $F$ ). If there are matter fields which are not simple fluids with $p(\rho)-$ for example an imperfect fluid possessing a bulk viscosity or a gas of free particles with anisotropic pressures - then additional parameters are required to specify them. There can still be overall constraints - a trace-free energy-momentum tensor, for example, in the cases of electric and magnetic fields or Yang-Mills fields - and we would just count the number of independent terms in the total energy-momentum tensor [51].

In the case of the Planck or WMAP mission data analyses, 6 constants are chosen to define the standard (minimal) $\Lambda C D M$ model. For WMAP [43, these are the present-day Hubble expansion rate, $H_{0}$, the densities of baryons and cold dark matter, the optical depth, $\tau$, at a fixed redshift, and the amplitude and slope of an assumed power-law spectrum of curvature inhomogeneities on a specified reference length scale. This is equivalent to including three matter fields (radiation, baryons, cold dark matter) but the standard $\Lambda C D M$ assumes 
zero spatial curvature, $k$, ab initio so a relaxation of this would add a curvature term or a dark energy field, because when $k \neq 0$ the latter could no longer be deduced from the other densities and the critical density (defined by $H_{0}$ ). The light neutrino densities are assumed to be calculable from the radiation density using the standard cosmological thermal history, so there are effectively $F=5$ matter fields (with $k$ set to zero in the base model) and a metric time derivative determined by $H$ ). All deviations from isotropy and homogeneity enter only at the level of perturbation theory and are characterised by the spectral amplitude and slope on large scales; the amplitude on small scales ('acoustic peaks' in the power spectrum) is determined from that on large scales by an $e^{-2 \tau}$ damping factor determined by the optical depth parameter $\tau$. The Planck mission parameter choice is equivalent to this 44.

Although a general solution of the Einstein equations requires the full complement of arbitrary functions, different parts of the general solution space can have behaviours of quite different complexity. For example, when $N \leq 9$ there are homogeneous vacuum universes which are dynamically chaotic [29, 52] but the chaotic behaviour disappears when $N \geq 10$ even though the number of arbitrary constants remains maximal for each $N$ [53]. Hence, the dynamical complexity can fail to be captured by the function-counting approach.

\subsection{Einstein's 'strength'}

As an interesting historical aside, we should mention Einstein's attempt to study the power of mathematical formalism to describe physical theories by ascribing to them a numerical measure of their predictive power, which he called the 'strength' of a system of differential equations. It was to be measured by the number of free pieces of initial data needed to determine the general solutions of the equations. Einstein believed that 'The smaller the number of free data consistent with the system of field equations, the 'stronger' is the system. It is clear that in the absence of any other viewpoint from which to select the equations, one will prefer a 'stronger' system to a less strong one.' 54] This was the method Einstein proposed to follow in his quest for a unified field theory (how different to the methodology that led to all his past great successes). The enumeration of the strength of a system of equations for $d$ variables began by expanding an analytic function of these variables in a Taylor about a point and noting that at $n^{\text {th }}$ order the total number of terms in the expansion is

$$
\left(\begin{array}{c}
n+d-1 \\
n
\end{array}\right) \equiv \frac{(n+d-1) !}{n !(d-1) !} .
$$

If there are field equations which ensure that when the function is specified arbitrarily on a $d-1$ dimensional (spatial) surface then those in the remaining (temporal) dimension are determined by them, then only $\left(\begin{array}{c}d+1 \\ n\end{array}\right)$ of the Taylor series coefficients remain arbitrary. The fraction of coefficients that remain free is therefore 


$$
\frac{\left(\begin{array}{c}
d-1 \\
n
\end{array}\right)}{\left(\begin{array}{c}
d \\
n
\end{array}\right)}=\frac{d-1}{n+d-1} .
$$

In the case of Einstein's equations we have coordinate covariances and constraint equations to use to reduce the count of free functions. The resulting strength turns out to be identical to the count of independent pieces of initial data for the metric and its first derivative that we have just described, giving a strength of 4 in vacuum. A similar count can be done for Maxwell's equations (which have the same strength), or other equations of mathematical physics. A fuller discussion is given in refs [55, 56.

\section{$5 \quad$ More general gravity theories}

There has been considerable interest in trying to explain the dark energy as a feature of a higher-order gravitational theory that extends the lagrangian of general relativity in a non-linear fashion [57, 58, 55, 60. This offers the possibility of introducing a lagrangian that is a function of $L=f\left(R, R_{a b} R^{a b}\right)$ of the scalar curvature $R$ and/or the Ricci scalar $R_{a b} R^{a b}$ in anisotropic models, with the property that it contributes a slowly varying dark energy-like behaviour at late times without the need to specify an explicit cosmological constant. However, these higher-order lagrangian theories (excluding the Lovelock lagrangians in which the variation of the higher-order terms contribute pure divergences 61 . and so the field equations are always 2 nd order in any spatial dimension) all have 4th-order field equations in 3-dimensional space when $f \neq A+B R$, with $A, B$ constants. This means that the initial data set for such theories is considerably enlarged because we must specify $\ddot{g}_{\alpha \beta}$ and $\dddot{g}_{\alpha \beta}$ in addition to $g_{\alpha \beta}$ and $\dot{g}_{\alpha \beta}$. In $N$ space dimensions, this results in a further $N(N+1)$ functions of $N$ variables and so a general cosmological model with $F$ fluids and $S$ scalar fields requires a specification of $2\left(N^{2}-1\right)+F(N+1)+2 S=(N+1)(F+2 N-2)$ $+2 S$ independent arbitrary spatial functions. For $N=3$, this is $16+4 F+2 S$. General relativity with 4 matter fields plus a cosmological constant requires 20 spatial functions plus one constant, in general, whereas a higher-order gravity theory with 4 matter fields and no scalar fields (and no cosmological constant because it should presumably emerge from the metric behaviour) requires the specification of 32 spatial functions.

\section{$6 \quad$ Reducing functions to constants}

The commonest simplification used to reduce the size of the cosmological characterisation problem is to turn the spatial functions into constants. This simplification will be an exact if the universe is assumed to be spatially homogeneous. The set of possible spatially homogeneous and isotropic universes with natural 
topology is based upon the classification of homogeneous 3-spaces created by Bianchi [62, 63, 64, 65] (together with the exceptional case of Kantowski-SachsKompanyeets-Chernov with $S^{1} \times S^{2}$ topology [66, 67] which we will ignore here as it displays non-generic behaviour).

The most general Bianchi type universes are those of types $V I_{h}, V I I_{h}, V I I I$ and $I X$. Of these, only types $V I I_{h}$ and $I X$, respectively, contain open and closed isotropic Friedmann subcases. These most general Bianchi types are all defined by 4 arbitrary constants in vacuum plus a further 4 for each noninteracting perfect fluid source. Therefore, in 3-dimensional spaces, the most general spatially homogeneous universes containing $F$ fluids are defined by $4(1+$ $F)$ arbitrary constants. This suggests that they might be the leading order term in a linearisation of the general inhomogeneous solution in the homogeneous limit. However, things might not be so simple. The 4 -function space of solutions to Einstein's models like type $I X$ with compact spaces has a conical structure at points with Killing vectors and so linearisation about the points must control an infinite number of spurious linearisations (associated with all the tangents that can be drawn through the point of the cone but don't run down the side of the cone) that are not the leading-order terms in any convergent series expansion of a true solution [68, 11].

The Bianchi classification of spatially homogeneous universes derives from the classification of the group of isometries with 3-dimensional subgroups that act simply transitively on the manifold. Intuitively, these give cosmological histories that look the same to observers in different places on the same hypersurface of constant time.

The Bianchi types are subdivided into two classes 69]: Class A contains types $I(1+F), I I(2+3 F), V I_{0}(3+4 F), V I I_{0}(3+4 F), V I I I(4+4 F)$ and $I X(4+$ $4 F)$, while Class B contains types $V(1+4 F), I V(3+4 F), I I I(3+4 F), V I_{-1 / 9}(4+$ $3 F), V I_{h}(4+4 F)$ and $V I I_{h}(4+4 F)$. The brackets following each Roman numeral of the Bianchi type geometry contain the number of constants defining the general solution when $F$ non-interacting perfect fluids, each with $p>-\rho$, are present, so $F=0$ defines the vacuum case. For example, Bianchi type I denoted by $I(1+F)$ is defined by one constant in vacuum (when it is the Kasner metric) and one additional constant for the value of the density when each matter field is added. For simplicity, we have ignored scalar fields here, but to include them simply add $2 S$ inside each pair of brackets. The Euclidean metric geometry in the type $I$ case requires $R_{0 \alpha}=0$, identically, and so the 3 non-comoving velocities (and hence any possible vorticity) must be identically zero. This contains the zero-curvature Friedmann model as the isotropic (zero parameter) special case. In the next simplest case, of type $V$, the general vacuum solution was found by Saunders [70] and contains one parameter, but each additional perfect-fluid adds 4 parameters because it requires specification of a density and three non-zero $u_{\alpha}$ components. The spatial geometry is a Lobachevsky space of constant negative isotropic curvature. The isotropic subcases of type $V$ are the zero-parameter Milne universe in vacuum and the $F$-parameter open Friedmann universe containing $F$ fluids.

In practice, one cannot find exact homogeneous general solutions contain- 
ing the maximal number of arbitrary constants because they are too complicated mathematically, although the qualitative behaviours are fairly well understood, and many explorations of the observational effects use the simplest Bianchi I or V models (usually without including non-comoving velocities) because they possess isotropic 3-curvature and add only a simple fast-decaying anisotropy term (requiring one new constant parameter) to the Friedmann equation. The most general anisotropic metrics which contain isotropic special cases, of types $V I I$ and $I X$, possess both expansion anisotropy (shear) and anisotropic three-curvature. Their shear falls off more slowly (logarithmically in time during the radiation era) and the observational bounds on it are much weaker [36], ,71], [72, ,73], ,35], 74].

\section{Some effects of the topology of the universe}

So far, we have assumed that the cosmological models in question have the 'natural' topology, that is $R^{3}$ for the 3-dimensional flat and negatively curved spaces and $S^{3}$ for the closed spaces. However, compact topologies can also be imposed upon flat and open universes to make their spatial volumes finite and there has been considerable interest in this possibility and its observational consequences for optical images of galaxies and the CMB, [75, 76, 77].

The classification of compact negatively-curved spaces is a challenging mathematical problem. When compact spatial topologies are imposed on spatially flat and open homogeneous cosmologies it produces a major change in their relative generalities and the numbers of constants needed to specify them in general.

The most notable consequences of a compact topology on 3-dimensional homogeneous spaces is that the Bianchi universes of types $I V$ and $V I_{h}$ no longer exist at all and open universes of Bianchi types $V$ and $V I I_{h}$ must be isotropic with spaces that are quotients of a space of constant negative curvature, as required by Mostow's Rigidity theorem [78, 79, 80, 81, 82. The only universes with non-trivial structure that differs from that of their universal covering spaces are those of Bianchi types $I, I I, I I I, V I_{0}, V I I_{0}$ and VIII. The numbers of parameters needed to determine their general cosmological solutions when $F$ non-interacting fluids are present and the spatial geometry is compact are now given by $I(10+F), I I(6+3 F), I I I\left(2+N_{m}^{\prime}+F\right), V I_{0}(4+4 F), V I I_{0}(8+4 F)$ and $V I I I\left(4+N_{m}+4 F\right)$, again with $F=0$ giving the vacuum case, as before, and an addition of $2 S$ to each prescription if $S$ scalar fields are included. Here, $N_{m}$ is the number of moduli degrees of freedom which measures of the complexity of the allowed topology, with $N_{m} \equiv 6 g+2 k-6 \equiv N_{m}^{\prime}-2 g$, where $g$ is the genus and $k$ is the number of conical singularities of the underlying orbifold [80, 81]. It can be arbitrarily large.

The rigidity restriction that compact types $V$ and $V I I_{h}$ must be isotropic means that compactness creates general parameter dependencies of $V(F)$ and $V I I_{h}(F)$ which are the same as those for the open isotropic Friedmann universe, or the Milne universe in vacuum when $F=0$. 
The resulting classification is shown in Table 1, 83. We see that the introduction of compact topology for the simplest Bianchi type $I$ spaces produces a dramatic increase in relative generality. Indeed, they become the most general vacuum models by the parameter-counting criterion. An additional 9 parameters are required to describe the compact type $I$ universe compared to the case with non-compact Euclidean $R^{3}$ topology. The reason for this increase is that at any time the compact 3-torus topology requires 3 identification scales in orthogonal directions to define the torus and 3 angles to specify the directions of the vectors generating this lattice plus all their time-derivatives. This gives 12 parameters, of which 2 can be removed using a time translation and the single non-trivial Einstein constraint equation, leaving 10 in vacuum compared to the 1 required in the non-compact Kasner vacuum case.

The following general points are worth noting:

(i) The imposition of a compact topology changes the relative generalities of homogeneous cosmologies;

(ii) The compact flat universes are more general in the parameter-counting sense than the open or closed ones;

(iii) Type VIII universes, which do not contain Friedmann special cases but can in principal become arbitrarily close to isotropy are the most general compact universes.

The most general case that contains an isotropic special case is that of type $V I I_{0}$ - recall that the $V I I_{h}$ metrics are forced to be isotropic so open Friedmann universes now become asymptotically stable 80 and approach the Milne metric whereas in the non-compact case they are merely stable and approach a family of anisotropic vacuum plane waves [37. This peculiar hierarchy of generality should be seen as a reflection of how difficult it is to create compact homogeneous spaces supporting these homogeneous groups of motions.

Table 1: The number of independent arbitrary constants required to prescribe the general 3-dimensional spatially homogeneous Bianchi type universes containing $F$ perfect fluid matter sources in cases with non-compact and compact spatial topologies. The vacuum cases arise when $F=0$. If $S$ scalar fields are also present then each parameter count increases by $2 S$. The type IX universe does not admit a non-compact geometry and compact universes of Bianchi types $I V$ and $V I_{h}$ do not exist. Types III and VIII have potentially unlimited topological complexity and arbitrarily large numbers of defining constants parameters through the unbounded topological parameters $N_{m} \equiv 6 g+2 k-6$ and $N_{m}^{\prime}=N_{m}+2 g$, where $g$ is the genus and $k$ is the number of conical singularities of the underlying orbifold [83]. 


\begin{tabular}{|c|c|c|}
\hline Cosmological & \multicolumn{2}{|c|}{ No. of defining parameters with $F$ non-interacting fluids } \\
\cline { 2 - 3 } Bianchi Type & Non-compact topology & Compact topology \\
\hline$I$ & $1+F$ & $10+F$ \\
\hline$I I$ & $2+3 F$ & $6+3 F$ \\
\hline$V I_{0}$ & $3+4 F$ & $4+4 F$ \\
\hline$V I I_{0}$ & $3+4 F$ & $8+4 F$ \\
\hline$V I I I$ & $4+4 F$ & $4+N_{m}+4 F$ \\
\hline$I X$ & - & $4+4 F$ \\
\hline$I I I$ & $3+4 F$ & $2+N_{m}^{\prime}+F$ \\
\hline$I V$ & $3+4 F$ & - \\
\hline$V$ & $1+4 F$ & $F$ \\
\hline$V I_{h}$ & $4+4 F$ & - \\
\hline$V I I_{h}$ & $4+4 F$ & $F$ \\
\hline
\end{tabular}

\section{Inhomogeneity}

The addition of inhomogeneity turns the constants defining the cosmological problem into functions of three space variables. For example, we are familiar with the linearised solutions for small density perturbations of a Friedmann universe with natural topology which produces two functions of space that control temporally growing and decaying modes. The function of space in front of the growing mode is typically written as a power-law in length scale (or wave number) and so has arbitrary amplitude and power index (both usually assumed to be scale-independent constants to first or second order) which can fitted to observations. Clearly there is no limit to the number of parameters that could be introduced to characterise the density inhomogeneity function by means of a series expansion around the homogeneous model (and the same could be done for any vortical or gravitational-wave perturbation modes) but the field equations would leave only 8 independent functions. Further analysis of the function characterising the radiation density is seen in the attempts to measure and calculate the deviation of its statistics from gaussianity [84] and to reconstruct the past light-cone structure of the universe [85]. Any different choice of specific spatial functions to characterise inhomogeneity in densities or gravitational waves requires some theoretical motivation. What happens in the inhomogeneous case if open or flat universes are given compact spatial topologies is not known. As we have just seen, the effects of topology on the spatially homogeneous anisotropic models was considerable whereas the effects on the overall evolution of isotropic models (as opposed to the effects on image optics) is insignificant. It is generally just assumed that realistically inhomogeneous universes with non-positive curvature (or curvature of varying sign) can be endowed with a compact topology and, if so, this places no constraints on their dynamics. However, both assumptions would be untrue for homogeneous universes and would necessarily fail for inhomogeneous ones in the homogeneous limit. it remains to be determined what topological constraints arise in the inhomogeneous cases. They could be weaker because inhomogeneous anisotropies can be local (far small in 
scale than the topological identifications) or they could be globally constrained like homogeneous anisotropies. Newtonian intuitions can be dangerous because compactification of a Newtonian Euclidean cosmological space seems simple but if we integrate Poisson's equation over the compact spatial volume we see that the total mass of matter must be zero. This follows from Poisson's equation since

$$
0=\int_{V} \nabla^{2} \Phi d V=4 \pi G \int_{V} \rho d V=4 \pi G M,
$$

where $V$ is the compact spatial volume, $M$ the total mass, and $\Phi$ is the Newtonian gravitational potential.

In practice, there is a divide between the complexity of inhomogeneity in the universe on small and large scales. On large scales there has been effectively no processing of the primordial spectrum of inhomogeneity by damping or non-linear evolution. Its description is well approximated by replacing a smooth function by a power-law defined by 2 constants, as for the microwave background temperature fluctuation spectrum or the 2-point correlation function of galaxy clustering. Here, the defining functions may be replaced by statistical distributions for specific features, like peak or voids in the density distribution. On small scales, inhomogeneities that entered the horizon during the radiation era can be damped out by photon viscosity or diffusion and may leave distortions in the background radiation spectrum as witness to their earlier existence. The baryon distribution may provide baryon acoustic oscillations which yield potentially sensitive information about the baryon density 43, 44. On smaller scales that enter the horizon later, where damping and non-linear self-interaction has occurred, the resulting distributions of luminous and dark matter are more complicated. However, they are correspondingly more difficult to predict in detail and numerical simulations of ensembles of models are used to make predictions down to the limit of reliable resolution. Predicting their forms also requires a significant extension of the simple, purely cosmological enumeration of free functions that we have discussed so far. Detailed physical interactions, 3-d hydrodynamics, turbulence, shocks, protogalaxy shapes, magnetic fields, and collision orientations, all introduce additional factors that may increase the parameters on which observable outcomes depend. The so called bias parameter, equal to the ratio of luminous matter density to the total density, is in reality a spatial function that is being used to follow the ratio of two densities because one (the dark matter) is expected to be far more smoothly distributed than the other. All these small scale factors combine to determine the output distribution of the baryonic and non-baryonic density distributions and their associated velocities.

\subsection{Links to Observables}

The free spatial functions (or constants) specifying inhomogeneous (homogeneous) metrics have simple physical interpretations. In the most general cases the 4 vacuum parameters can be thought of as giving two shear modes (ie 
time-derivatives of metric anisotropies) and two parts of the anisotropic spatial curvature (composed of ratios and products of metric functions). In the simplest vacuum models of type $I$ and $V$ the three-curvature is isotropic an there is only one shear parameter. It describes the allowed metric shear and in the type $V$ model a second parameter is the isotropic three-curvature (which is zero in type $I$ ) - just like $k$ in the Friedmann universe models. When matter is added there is always a single $\rho$ (or $p$ ) for each perfect fluid and up to three non-comoving fluid velocity components. If the fluid is comoving, as in type $I$ only the density parameter is required for each fluid; in type $V$ there can also be 3 non-comoving velocities. The additional parameters control the expansion shear anisotropy, anisotropic 3-curvature. They may all contribute to temperature anisotropy in the $\mathrm{CMB}$ radiation but the observed anisotropy is determined by an integral down the past null cone over the shear (effectively the shear to Hubble rate ratio at last scattering of the $\mathrm{CMB}$ ), rather than the Weyl curvature modes driven by the curvature anisotropy (which can be oscillatory [86, and so can be periodically be very small even though the envelope is large), while the velocities contribute dipole variations. Thus, it is difficult to extract complete information about all the anisotropies from observations of the lower multipoles of the CMB alone in the most general cases [87, 85, 88].

At present, the observational focus is upon testing the simplest possible $\Lambda C D M$ model, defined by the smallest number (six) of parameters. As observational sensitivity increases it will become possible to place specific bounds or make determinations of the full spectrum of defining functions (or constants), or at least to confirm that they remain undetectably small as inflation would lead us to expect. In an inflationary model they can be identified with the spatial functions defining the asymptotic expansion around the de Sitter metric [8].

There have also been interesting studies of the observational information needed to determine the structure of our past null cone rather than constanttime hypersurfaces in the Universe [89, extending earlier investigations of the links between observables and general metric expansions by McCrea [90] and by Kristian and Sachs [91].

The high level of isotropy in the visible universe, possibly present as a consequence of a period of inflation in the early universe [6], or special initial conditions [93, 35, 50, 94, 95, is what allows several of the defining functions of a generic cosmological model to be ignored on the grounds that they are too small to be detected with current technology. An inflationary theory of the chaotic or eternal variety, in which inflation only ends locally, will lead to some complicated set of defining functions that exhibit large smooth isotropic regions within a complicated global structure which is beyond our visual horizon and unobservable (although not necessarily falsifiable within a particular cosmological model). However, despite the success of simple cosmological theories in explaining almost all that we see in the universe, it is clear that there is an under-determination problem: we cannot make enough observations to specify the structure of space-time and its contents, even on our past light cone, let alone beyond it. It is not a satisfactory methodology to use observations to construct a description of space-time. Rather, we proceed by creating parametrised 
descriptions that follow from solutions of Einstein's equations (or some other theory) and then constrain the free parameters by using he observational data. Despite the widespread lip-service paid to Popper's doctrine of falsification as a scientific methodology, its weaknesses are especially clear in cosmology. It assumes that all observations and experimental results are correct and unbiased - that what you see is what you get. In practice, they are not and you never know whether observational data is falsifying a theory, or is based on wrong measurements, or subject to some unsuspected selection effect [96]. All that observational science can ever do is change the likelihood of a particular theory being true or false. Sometimes the likelihood can build up (or down) to such an extent that we regard a theory being tested (like the expansion of the universe) as 'true' or (like cold fusion) as 'false'.

Acknowledgements. Support from the STFC (UK) and the JTF OxfordCambridge Philosophy of Cosmology programme grant is acknowledged.

\section{References}

[1] A. Einstein, S. -B. Preuss. Akad. Wiss. p.142 (1917)

[2] A. S. Eddington, Mon. Not Roy. astron. Soc. 90, 668 (1930) E. R. Harrison, Rev. Mod. Phys. 39, 862 (1967); G.W. Gibbons, Nucl. Phys. B 292, 784 (1987); ibid., 310, 636 (1988); J.D. Barrow, G.F.R. Ellis, R. Maartens and C. Tsagas, Class. Quant. Grav. 20, L155 (2003)

[3] S.W. Hawking and G.F.R. Ellis,Hawking SW and Ellis GFR, The Large Scale Structure of Space-Time, (Cambridge University Press, Cambridge, 1973) Hawking SW and Penrose R., Proc. R. Soc. London A 314, 529 (1970)

[4] K. Gödel, Rev. Mod. Phys. 21, 447 (1949)

[5] C.W. Misner, Phys. Rev. Letts. 19, 533 (1967) and Ap. J. 151, 431 (1968)

[6] A.H. Guth, Phys. Rev. D 23, 347 (1980)

[7] R.M. Wald, Phys. Rev. D 28, 2118 (1982); J.D. Barrow, in The Very Early Universe, ed. G.W. Gibbons, S.W. Hawking \& S.T.C. Siklos, (Cambridge U. P., Cambridge,1983) pp. 267-272; W. Boucher and G.W. Gibbons, ibid pp. 273-8; L. Jensen and J. Stein Schabes, Phys. Rev. D 35, 1146 (1987); J.D. Barrow, Phys. Lett. B 187,12 (1987).

[8] A.A. Starobinsky, Sov. Phys. JETP Lett. 37, 66 (1983)

[9] R. Earman, Bangs, Crunches, Whimpers and Shrieks: singularities and acausalities in relativistic spacetimes, (Oxford U.P., Oxford, 1995)

[10] S.W. Hawking, Gen. Rel. Gravitation 1, 393 (1971)

[11] J.D. Barrow and F.J. Tipler, Phys. Reports 56, 371 (1979) 
[12] J.D. Barrow and G. Götz, Class. Quantum Gravity, 6, 1253 (1989)

[13] J.D. Norton, in The Expanding Worlds of General Relativity, eds. H. Goenner, J. Renn, J. Ritter, and T. Sauer, Einstein Studies vol. 7, 271-323 (1999)

[14] E.A. Milne and W.H. McCrea, Quart. J. Math. Oxford Ser. 5, 73 (1934).

[15] D. Saari and Z. Xia, Hamiltonian Dynamics and Celestial Mechanics, (Amer. Math. Soc., Providence RI,1996)

[16] G.F.R. Ellis, gr-qc/1107.3669

[17] Z. Xia, , Ann. Math.,135, 411 (1992)

[18] J.D. Barrow, The Infinite Book, (Jonathan Cape, London, 2005).

[19] J.D. Barrow and G.W. Gibbons, Mon. Not. Roy. Astron. Soc. 446, 3874 (2014)

[20] G. Lemaître, Mon. Not. Roy. astron. Soc. 91, 483 (1931)

[21] Einstein supposed said that introducing the cosmological constant was the biggest blunder of his life, presumably because it meant that he failed to predict the expansion of the universe, but I can find no primary trace of such a remark. Einstein did make a similar remark about his signing of the letter to Roosevelt urging the construction of an atomic bomb (although he didn't work on the Manhattan Project because he was judged to be a potential security risk). In 1954, he called his decision to sign 'the one great mistake in my life' (R. Clark, Einstein: the life and times, p. 752) which suggests he never made such a remark about the cosmological constant.

[22] A. Friedmann, Zeit f. Phys. 10, 377 (1922)

[23] G. Lemaître, Ann. Soc. Sc. Bruxelles, 53A, 51 (1933)

[24] S. Chandrasekhar and J.C. Miller, Mon. Not. Roy. astron. Soc. 167, 63 (1974)

[25] J.D. Barrow and F.J. Tipler, The Anthropic Cosmological Principle, (Oxford U.P., Oxford, 1986), p.612.

[26] A. Borde and A. Vilenkin, Phys. Rev. Lett. 72, 3305 (1994)

[27] E.M. Lifshitz and I.M. Khalatnikov, Adv. Phys. 12, 185 (1963)

[28] For an elementary account see J.D. Barrow, The Origin of the Universe, (Basic Bks. New York, 1993), chap.3.

[29] V.A. Belinskii, I.M. Khalatnikov and E.M. Lifshitz, Adv. Phys. 19, 525 (1970)

[30] R. B. Partridge and D.T. Wilkinson, Phys. Rev. Lett. 18, 557 (1967) 
[31] J.D. Barrow and R.M. Matzner, Mon. Not. Roy. astron. Soc. 181, 719 (1977)

[32] C.W. Misner, Phys. Rev. Lett. 22, 1071 (1969)

[33] A.G. Doroshkevich and I.D. Novikov, Sov. Astron. 14, 763 (1971)

[34] C.B. Collins and J.M.Stewart, Mon. Not. Roy. astron. Soc. 153, 419 (1971)

[35] J.D. Barrow, Phys. Rev. D 51, 3113 (1995)

[36] C.B. Collins and S.W. Hawking, Mon. Not. R. astron. Soc. 162, 307 (1972)

[37] J.D. Barrow and D.H. Sonoda, Phys. Reports 139, 1 (1986)

[38] J.D. Barrow, Quart. J. Roy. astron. Soc. 23, 344 (1982)

[39] F. Hoyle and J.V. Narlikar, Proc. Roy. Soc. A 273, 1 (1963)

[40] L. A. Kofman, A. D. Linde and V. F. Mukhanov, JHEP 0210, 057 (2002); G.W. Gibbons and N. Turok, Phys. Rev. D 77, 063516 (2008); A. Corichi and D. Sloan, arXiv:1310.6399.

[41] M. Rowan Robinson, The Nine Numbers of the Cosmos, (Oxford U.P., Oxford,1999).

[42] M.J. Rees, Just Six Numbers: the deep forces that shape the universe, (Phoenix, London, 2001).

[43] D.N. Spergel et al (WMAP), Ap. J. Supplt. 148,175, (2003).

[44] P.A.R. Ade et al (Planck Collaboration Paper XVI), arXiv:1303.5076.

[45] J.D. Barrow and D. Shaw, Phys. Rev. Lett. 106, 101302 (2011)

[46] L. Landau and E.M. Lifshitz, The Classical Theory of Fields, 4th rev. edn. (Pergamon, Oxford, 1975)

[47] J.D. Barrow, Gravitation and Hot Big Bang Cosmology, In The Physical Universe: The Interface Between Cosmology, Astrophysics and Particle Physics', eds. J.D. Barrow, A. Henriques, M. Lago \& M. Longair, pp.1-20, (Springer-Verlag, Berlin,1991).

[48] J.D. Barrow, Class. Quant. Grav. 21, L79 (2004); J.D. Barrow and A.A.H. Graham, arXiv: 1501.04090

[49] J.D. Barrow, S. Cotsakis and A. Tsokaros, Class. Quant. Grav. 27, 165017 (2010)

[50] M. Heinzle and P. Sandin, Comm. Math. Phys. 313, 385 (2012)

[51] J.D. Barrow, Phys. Rev. D 89, 064022 (2014) 
[52] J.D. Barrow, Phys. Rev. Lett. 46, 963 (1981) and Phys. Reports 85, 1 (1982); D. Chernoff and J.D. Barrow, Phys. Rev. Lett. 50, 134 (1983)

[53] J. Demaret, M. Henneaux and P. Spindel, Phys. Lett. 164, 27 (1985)

[54] A. Einstein, The Meaning of Relativity, Appendix II, p. 136, 6th rev. edn. (Methuen, London and NY,1956)

[55] B. Schutz, J. Math. Phys. 16, 855 (1975)

[56] K.H. Mariwalla, J. Math. Phys. 15, 468 (1974)

[57] J.D. Barrow and A.C. Ottewill, J. Phys. A 16, 2757 (1983)

[58] V. Faraoni and T. Sotiriou, Rev. Mod. Phys. 82, 451(2010)

[59] T. Clifton, P. G. Ferreira, A. Padilla, C. Skordis, Phys. Reports 513, 1 (2012)

[60] T. Clifton and J.D. Barrow, Phys. Rev. D 72,123003 (2005)

[61] D. Lovelock, J. Math. Phys. 12, 498 (1971)

[62] L. Bianchi, Mem. Matematica Fis. d. Soc. Ital. delle Scienza, Ser. Terza 11, 267 (1898) reprinted in Gen. Rel. Grav. 33, 2171 (2001)

[63] A.H. Taub, Ann. Math. 53, 472 (1951)

[64] M.A.H. MacCallum, in General Relativity: An Einstein Centenary Survey, eds. S.W. Hawking and W. Israel, (Cambridge UP, Cambridge, 1979), pp.533-576.

[65] G.F.R. Ellis, S.T.C. Siklos and J. Wainwright, in Dynamical Systems in Cosmology, eds. J. Wainwright and G.F.R. Ellis (Cambridge UP, Cambridge, 1997), pp. 11-42.

[66] R. Kantowski and R.K. Sachs, J. Math. Phys. 7, 443(1966)

[67] A.S. Kompaneets and A.S. Chernov, Sov. Phys. JETP 20,1303 (1964)

[68] A.E. Fischer, J.E. Marsden and V. Moncrief, Ann. Inst. H. Poincaré 33,147 (1980)

[69] G.F.R. Ellis and M.A.H. MacCallum, Comm. Math. Phys. 12,108 (1969)

[70] P.T. Saunders, Mon. Not. R. astron. Soc.142, 213 (1969)

[71] A.G. Doroshkevich, V. Lukash and I.D. Novikov, Sov. Phys. JETP 37, 739 (1973)

[72] J.D. Barrow, Mon. Not. R. astron. Soc. 175, 359 (1976)

[73] J.D. Barrow, R. Juszkiewicz and D.N. Sonoda, Mon. Not. R. astron. Soc. 213, 917 (1985) 
[74] J.D. Barrow, Phys. Rev. D 55, 7451 (1997)

[75] G.F.R. Ellis, Gen. Rel. Gravn. 2, 7 (1971)

[76] R. Aurich, S. Lustig, F. Steiner and H. Then, Class. Quant. Grav. 21, 4901 (2004)

[77] P.A.R. Ade et al (Planck Collaboration Paper XXVI), arXiv:1303.5086

[78] A. Ashtekar and J. Samuel, Class. Quant. Grav. 8, 2191 (1991)

[79] H.V. Fagundes, Gen. Rel. Gravn. 24, 199 (1992)

[80] J.D. Barrow and H. Kodama, Int. J. Mod. Phys. D 10, 785 (2001)

[81] J.D. Barrow and H. Kodama, Class. Quant. Grav.18,1753 (2001)

[82] H. Kodama, Prog. Theor. Phys. 107, 305 (2002)

[83] J.D. Barrow, Phys. Rev. D 89, 064022 (2014)

[84] P.A.R. Ade et al (Planck Collaboration Paper XXIV), arXiv:1303.5084

[85] R. Maartens, Phil. Trans. R. Soc. A 369, 5115 (2011)

[86] W.C. Lim, U.S. Nilsson and J. Wainwright, Class. Quant. Grav. 18, 5583 (2001)

[87] W. Stoeger, M. Araujo, T. Gebbie, Ap. J. 476, 435 (1997)

[88] U.S. Nilsson, C. Uggla, J. Wainwright and W. C. Lim, Ap. J. 522, L1 (1999)

[89] G.F.R. Ellis , S.D. Nell, R. Maartens, W.R. Stoeger and A.P. Whitman, Phys., Rep. 124, 315 (1985)

[90] W.H. McCrea, Zeit. Astrophys. 9, 290 (1934) and Zeit. Astrophys.18, 98 (1939), reprinted in Gen. Rel. Grav. 30, 315 (1998)

[91] J. Kristian and R.K. Sachs, Ap. J. 143, 379 (1966)

[92] A. Guth, Phys. Rev. D 23, 347 (1981)

[93] J.D. Barrow, Nature 272, 211 (1978)

[94] R. Penrose, Cycles of Time, (Bodley Head, London, 2010); P. Tod, arXiv:1309.7248

[95] J. Hartle and S.W. Hawking, Phys. Rev. D 28, 2960 (1983)

[96] J.D. Barrow, in Seeing Further: The Story of science and the Royal Society, Royal Society 350th Anniversary Volume, ed. B. Bryson, (Harper Collins, London, 2010), p. 361-384. 